\title{
Controle de vibrações em vigas usando buracos prescritos e atuadores piezoelétricos
}

\section{Control of vibrations in beams using prescribed holes and piezoelectric actuators}

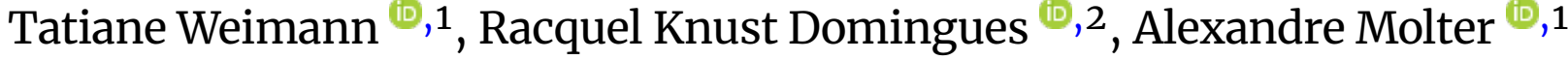 \\ ${ }^{1}$ Universidade Federal de Pelotas, ${ }^{2}$ Universidade Federal de Santa Catarina \\ *tatiane.weimann@ufpel.edu.br; racquel.knust@posgrad.ufsc.br; alexandre.molter@ufpel.edu.br
}

Recebido: 29/06/2021. Revisado: 29/06/2021. Aceito: 05/07/2021.

\begin{abstract}
Resumo
A implementação de métodos de controle de vibrações é cada vez mais necessária quando se trata da utilização de estruturas leves e flexíveis nos projetos mecânicos. O surgimento de vibrações em estruturas, mediante movimento, sempre merece a atenção dos projetistas, pois as mesmas, podem ser indesejadas quando afetam a vida útil da estrutura ou danificam o seu entorno. Com isso, este trabalho propõe o uso de dois tipos de controle numa viga engastada em um dos lados. A primeira técnica aplicada foi a de buracos prescritos, que é um tipo de controle passivo, onde é feita a retirada de material da estrutura de forma que ela fique com buracos. A vibração, quando entra neste buraco, fica presa em seu interior, não refletindo a onda para o restante da viga. A segunda técnica aplicada foi a implementação de um Regulador Quadrático Linear (LQR) para operar um atuador piezoelétrico. A formulação e modelagem da estrutura foi feita com base no Método dos Elementos Finitos (MEF), sendo utilizado para a discretização do domínio da estrutura. As vibrações foram analisadas por meio de análise modal. Foram feitas comparações da amplitude de vibração para o primeiro e segundo modos, considerando um, dois e três buracos prescritos na viga. Foram realizadas simulações computacionais para mostrar a eficiência das técnicas de controle propostas. Os resultados destas simulações são apresentados e discutidos.
\end{abstract}

Palavras-Chave: Controle de Vibrações; Buracos prescritos; Método dos Elementos Finitos; Regulador Quadrático Linear.

\begin{abstract}
The implementation of vibration control methods is increasingly necessary when it comes to the use of flexible structures in mechanical projects. The appearance of vibrations in structures, through movement, always deserves the attention of the designers, as they can be unwanted when they affect the structure's useful life or damage its surroundings. With that, this work offers the use of two types of control in a beam fixed on one side. The first technique applied was prescribed holes, which is a type of passive control where material is removed from the structure so that it has holes. The vibration, when it enters this hole, is trapped inside, not reflecting the wave to the rest of the beam. The second applied technique was the implementation of a Linear Quadratic Regulator (LQR) to operate a piezoelectric actuator. The formulation and modeling of the structure was made based on the Finite Element Method (FEM), being used for the discretization of the structure domain. The vibrations were analyzed through modal analysis. Vibration amplitude comparisons were made for the first and second modes, considering one, two and three prescribed holes in the beam. Computer simulations were carried out to show the efficiency of the proposed control techniques. The results of these simulations are presented and discussed.
\end{abstract}

Keywords: Vibration Control; Prescribed Holes; Finite Element Method; Linear Quadratic Regulator. 


\section{Introdução}

Cada vez mais o uso de estruturas leves e flexíveis se faz presente nos projetos de engenharia atuais. Isso se dá devido a diversos fatores, porém é possível destacar três como principais. Um deles é que a redução do peso de uma estrutura pode acarretar na redução do custo do projeto à qual ela faz parte. Um exemplo disso é quando reduzimos o peso de uma estrutura de um carro ou de um avião, fazendo com que o consumo de combustível para o transporte do mesmo reduza. Outro fator é a diminuição no custo operacional destes projetos, já que é usada uma quantidade menor de material. Outro fator ainda é quanto ao desempenho da estrutura, já que a diminuição do peso pode influenciar diretamente em características técnicas de um projeto, como, por exemplo, a aceleração de um veículo (Herrmann et al., 2018).

Porém o uso dessas estruturas pode acarretar em uma nova problemática para o projeto, as vibrações indesejadas. O baixo amortecimento e as baixas frequências naturais podem gerar movimentos vibratórios com altas amplitudes, que ocasionam diversos problemas para o projeto em questão (Dubay et al., 2014). Um deles é a falha estrutural que tem como consequência a diminuição da vida útil da estrutura em si. A irradiação de ruídos também é uma problemática bem comum relacionada às vibrações indesejadas. Além de interferências negativas no desempenho do projeto, como a falta de precisão ou a indução de vibrações no ambiente ao redor (Kelly, 2012).

Com isso, a necessidade de projetar essas estruturas juntamente com um controle de vibrações é essencial para um projeto eficiente. Existem diversas técnicas e arquiteturas para o controle de vibrações, que podem ser classificadas em controle passivo e controle ativo. A diferença básica entre esses dois tipos de controle é a necessidade ou não de inserção de energia no sistema para que haja a diminuição das vibrações (Nicoletti, 2013). O passivo é aquele onde não é necessário o uso de energia para que haja o controle, mas sim, são incorporados ao sistema dispositivos e/ou técnicas que absorvem, isolam ou dissipam a energia de vibração (Peña, 2017). Um dos dispositivos mais usados de controle passivo são os Amortecedores de Massa Sintonizados (AMS), que basicamente são massas adicionadas ao sistema que levam à modificação das características estruturais do mesmo. A consequência desta mudança é a dissipação da vibração presente no sistema pelo AMS, ou seja, o controle deste movimento vibratório (Chandran.P and Thampan, 2017). Uma outra forma de alterar as propriedades de uma estrutura é adicionando buracos com perfis pré-definidos em certos pontos estratégicos. Essa técnica de controle passivo tem sido amplamente usada nos últimos tempos já que tem um baixo custo para a sua implementação e pode gerar resultados relevantes para a atenuação de vibrações. Os buracos que atendem à uma lei de potência são um exemplo deste controle, onde sua inserção na estrutura faz com que a onda incidente na mesma, ao passar pelo buraco, tenha sua velocidade reduzida e sua amplitude aumentada. Ou seja, a vibração fica concentrada no interior do buraco, tendo uma parte muito pequena sendo refletida para o resto da estrutura (Pelat et al., 2020). Esta última técnica foi usada neste trabalho como controle passivo do sistema.
Muitas vezes, apenas o uso do controle passivo não é suficiente, sendo necessário usar o controle ativo em conjunto. Para este controle, em contrapartida, faz-se necessário o uso de energia. Geralmente, a proposta é gerar um movimento oscilatório contrário ao movimento presente na estrutura, para que, assim, haja o cancelamento da vibração, ou melhor, haja o controle deste movimento. Basicamente, a composição do controle ativo é um elemento para detectar a resposta de vibração (sensor), um elemento que vai receber este sinal de resposta e, a partir de alguma arquitetura implementada, gerar um sinal de controle (controlador), e um elemento que vai atuar com base nesse sinal de controle (atuador). Portanto, para se construir um controle ativo, primeiramente deve-se escolher uma arquitetura mais adequada para o controlador $\mathrm{e}$ um atuador eficiente para o sistema (Fuller et al., 1996).

Existem diversos tipos de atuadores usados nos sistemas de controle de vibrações em estruturas. Atuadores inerciais clássicos e modificados são exemplos deste uso. Estes atuadores são construídos com um ímã e uma bobina, conectadas à molas espirais, de tal forma que há o livre movimento destes componentes no sentido axial. Este movimento é conduzido conforme a polarização do conjunto ímã-bobina, gerando o movimento oscilatório necessário para o controle das vibrações (Kras and Gardonio, 2020). Recentemente, foi feita uma pesquisa em relação ao uso de atuadores de elastômeros dielétricos, que é um tipo de polímero que se contrai ou expande de acordo com a tensão elétrica aplicada à ele. Esse tipo de material tem um rápido tempo de resposta e, por isso, se mostrou hábil para o controle de vibrações (Hiruta et al., 2021). Um outro tipo de material amplamente usado neste tipo de controle é o piezolétrico. Ele também tem a característica de converter energia elétrica em energia mecânica e, por isso, se torna apropriado para este tipo de aplicação (Molter et al., 2018). Neste trabalho foi utilizado um atuador piezoelétrico para atuar no sistema.

A outra parte do controle ativo é a escolha da arquitetura a ser implementada no controlador. Existe uma grande diversidade de modelos e lógicas a disposição e, cada vez mais, surgem novas pesquisas e ideias nesse âmbito. Estudos recentes mostram que a aplicação de controladores não lineares, como um controlador baseado na lógica fuzzy, podem ser eficientes para compensar algumas imprecisões dos sensores, como ruídos e atrasos (Ramezani and Labafzadeh, 2021). Porém, as arquiteturas mais usadas para o controle de vibrações são as lineares. Uma das técnicas lineares usada para determinar o sinal de controle é a baseada na realimentação da velocidade. Nesse modelo, o sinal que opera o atuador é constituído de uma matriz de ganhos que multiplica o sinal de velocidade que vem do sensor do sistema. Por ter uma facilidade de implementação, esse tipo de controle é amplamente usado (Mohammadrezazadeh and Jafari, 2020). Porém, existem outros tipos de controladores lineares que têm a mesma facilidade de implementação e geram leis de controle mais precisas. Um deles é o Regulador Quadrático Ótimo (LQR). Nesse modelo o sinal de controle também é gerado com base na multiplicação de uma matriz de ganhos, porém, neste caso, esses ganhos são calculados com base na otimização de algum índice de desempenho do sistema, como, por exemplo, a energia interna do sistema ou a própria velocidade do sis- 
tema (Tian et al., 2020). Neste trabalho foi usado um LQR para controlar o atuador piezoelétrico.

Antes do desenvolvimento do problema de controle neste trabalho, foi necessário fazer a modelagem do movimento, que consiste em determinar o equacionamento matemático que o descreva. Geralmente, em problemas de mecânica contínua, essa função é obtida através da resolução de uma equação diferencial parcial. Porém, muitas vezes, essa resolução analítica pode se tornar uma tarefa complexa, dependendo da estrutura. Para isso, foram desenvolvidos diversos métodos que auxiliam neste processo. Um desses é o Método dos Elementos Finitos (MEF), que funciona como uma forma de discretizar o domínio geométrico da estrutura a ser modelada. $\mathrm{O}$ funcionamento básico consiste em dividir o domínio em pequenos elementos com uma geometria mais simples, onde, ao invés da modelagem ser feita considerando o movimento do contínuo, ela é feita aplicando as leis mecânicas aos elementos menores, considerando apenas seus deslocamentos pontuais. Isso nos leva à uma solução aproximada numérica de fácil obtenção (Liu and Quek, 2003).

Outras técnicas também foram usadas aliadas ao MEF, como a superposição modal e a representação em espaço de estados. A primeira delas é uma técnica de transformação de coordenadas do sistema que, utilizando o teorema da expansão, desacopla as equações do sistema, facilitando suas resoluções (Kelly, 2012). A segunda é uma técnica de representação das equações do sistema, que convertem as equações diferenciais de segunda ordem que compõem a modelagem de movimento de um sistema vibratório em um outro sistema, com o dobro de equações, porém de primeira de ordem (Ogata, 2014). Simulações computacionais são apresentadas para ilustrar os resultados obtidos no controle de vibrações.

\section{Modelagem de Elementos Finitos}

O MEF é comumente utilizado para resolver problemas mecânicos compostos por equações diferenciais parciais, no qual, a partir deste método o domínio da estrutura é discretizado em pequenos elementos mais simples (Liu and Quek, 2003). Dessa maneira, a ideia é analisar a equação de equilíbrio para cada elemento, obtendo a equação local, e posteriormente associar a contribuição de cada elemento em uma equação global.

O acoplamento destes elementos é feito através dos nós, que são os pontos onde os elementos se conectam. Os elementos discretizados são retangulares com quatro nós em cada elemento, no qual o domínio do elemento é denominado como $\Omega_{e}$. Em cada nó tem-se dois graus de liberdade, o movimento horizontal do nó $\left(x_{i}\right)$ e o movimento vertical do nó $\left(y_{i}\right)$, e ainda uma voltagem elétrica aplicada num elemento $\left(v_{a i}\right)$, como mostrado na Fig. 1.

Segundo Liu and Quek (2003), para uma análise dinâmica do elemento, a equação regente para um elemento pode ser descrita como:

$$
M_{e} \ddot{u}_{e}+C_{e} \dot{u}_{e}+K_{e} u_{e}=f_{e},
$$

sendo $u_{e}$ o vetor de deslocamento nodal do elemento, $f_{e} \mathrm{o}$ vetor de força, e as matrizes $M_{e}, K_{e}$ e $C_{e}$ a massa, rigidez

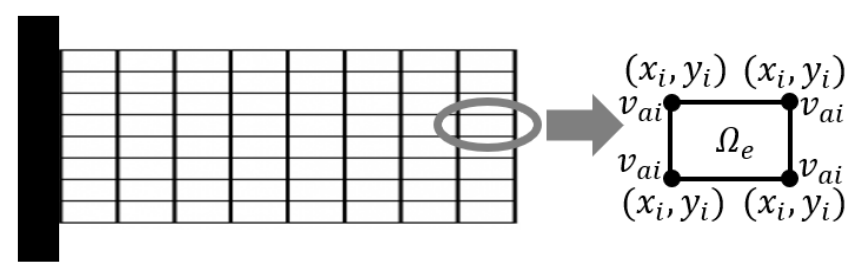

Figura 1: Domínio do projeto discretizado pelo MEF.

e amortecimento da estrutura, respectivamente. Estes vetores e matrizes são descritos da seguinte forma:

$$
\begin{aligned}
f_{e} & =\int_{\Omega_{e}} N^{\top} b_{e} d \Omega_{e}+\int_{\Gamma_{e}} N^{\top} t_{e} d \Gamma_{e}, \\
M_{e} & =\int_{\Omega_{e}} \rho N^{\top} N d \Omega_{e}, \\
K_{e} & =\int_{\Omega_{e}} B^{\top} c B d \Omega_{e}, \\
C_{e} & =\alpha K_{e}+\beta M_{e},
\end{aligned}
$$

onde $N$ é a matriz das funções interpoladoras, $b_{e}$ e $t_{e}$ são vetores que retratam as forças de corpo e as forças externas concentradas, respectivamente, $B$ é a matriz de relação entre a tensão e o deslocamento e $c$ é a matriz constitutiva do material. As contantes $\alpha$ e $\beta$ são determinadas de acordo com o modelo de Rayleigh e a constante $\rho$ é a densidade do material.

Neste trabalho foi considerado a análise das vibrações livres, isto é, os vetores de forças de corpo e de forças externas são nulos. Assim, a equação global dinâmica que rege sua estrutura é análoga à Eq. (1), podendo ser descrita como na Eq. (6):

$$
M \ddot{u}+C \dot{u}+K u=0 .
$$

As matrizes globais $M, C$ e $K$, continuam representando, respectivamente, a massa, amortecimento e rigidez, e são encontradas associando a contribuição de cada elemento da viga. $u$ é o vetor de deslocamentos nodais de toda estrutura.

Como neste trabalho também foi levado em conta um controle ativo, pode-se considerar o sinal de controle como uma força externa atuando no sistema que, neste caso, irá operar um atuador piezoelétrico. As equações constitutivas do material piezoelétrico são dadas por Hu et al. (2018):

$$
T=C S-e E, \quad D=e^{T} S+\epsilon E,
$$

onde $T$ são os componentes dos tensores mecânicos de tensão, $S$ são os componentes dos tensores mecânicos de deformação, $e$ são as constantes piezoelétricas, $\epsilon$ são as constantes dielétricas sob deformação mecânica, $D$ é o deslocamento elétrico (carga por unidade de área) e $E$ é o campo elétrico (tensão elétrica por unidade de comprimento).

Assume-se que os atuadores piezoelétricos estão isolados e o acoplamento é perfeito.

A equação global que rege a dinâmica estrutural deste 
trabalho pode ser descrita como:

$$
M \ddot{u}+C \dot{u}+K u=-K u v v_{a},
$$

onde $v_{a}$ é a tensão aplicada no atuador, como sinal de controle e este sinal é multiplicado pela matriz $K_{u v}$, expressa como:

$$
K_{u v}=\int_{\Omega_{d}} B^{T} e^{T} B_{v} d \Omega
$$

que é a matriz estrutural eletro-mecânica, sendo $B_{v}$ a matriz potencial de campo elétrico e $\Omega_{d}$ o espaço ocupado pelos atuadores (Hu et al., 2018).

\section{Modelagem da Análise Modal e representa- ção em Espaço de Estados}

De acordo com Gawronski (2004), a representação por coordenadas modais pode ser obtida pela transformação do modelo nodal. Dessa forma, com o intuito de facilitar a resolução da Eq. (8), usou-se a superposição modal para desacoplar as equações governantes do movimento, utilizando as coordenadas modais $\eta$. Essa modificação é realizada utilizando a matriz modal que é definida de acordo com a solução modal, resultando do problema de autovalor e autovetor descrito por:

$$
\left(K-\omega^{2} M\right) \Phi=0 .
$$

Os autovalores de $\omega$ encontrados, ou seja, as raízes da equação obtida após aplicar o determinante, são chamados de frequências naturais do sistema e são as frequências com que as vibrações livres ocorrem na estrutura. Já os autovetores associados aos autovalores encontrados $(\Phi)$, são denominados modos naturais que estão associados às frequências naturais. A matriz modal $\Phi$, é a matriz com todos os modos do sistema, entretanto, nas estruturas analisadas neste trabalho, apenas os primeiros modos contêm informações relevantes à análise do sistema. Sendo assim, utiliza-se a matriz modal truncada com $n$ modos.

Com a matriz modal truncada pode-se transformar $o$ vetor de deslocamento nodal em função das coordenadas modais (Becker et al., 2006), como:

$$
u=\Phi \eta \text {. }
$$

Substituindo a Eq. (11) na Eq. (8), obtém-se:

$$
M \Phi \ddot{\eta}+C \Phi \dot{\eta}+K \Phi \eta=-K_{u \nu} v_{a} .
$$

Multiplicando todos os termos da equação acima por $\Phi^{T}$, tem-se:

$$
\underline{M} \ddot{\eta}+\underline{C} \dot{\eta}+\underline{K} \eta=-\Phi^{T} K_{u v} v_{a},
$$

onde:

$$
\begin{aligned}
\underline{M} & =\Phi^{T} M \Phi=I, \\
\underline{C} & =\Phi^{T} C \Phi=Z, \\
\underline{K} & =\Phi^{T} K \Phi=\Omega .
\end{aligned}
$$

Como $\underline{M}=I$, a Eq. (13) pode ser reescrita como:

$$
\ddot{\eta}+Z \dot{\eta}+\Omega \eta=-\Phi^{T} K_{u v} v_{a},
$$

sendo:

$$
Z=\operatorname{diag}\left(2 \zeta_{i} \omega_{i}\right), \quad \Omega=\operatorname{diag}\left(\omega_{i}^{2}\right) .
$$

Considerando como variáveis de estado o deslocamento e a velocidade modal, $\xi=[\eta \dot{\eta}]^{T}$, pode-se reescrever a Eq. (15) na representação em espaço de estados como:

$$
\dot{\xi}=A \xi+B v_{a},
$$

sendo $v_{a}$ o vetor de entrada de controle do atuador, no qual:

$$
A=\left[\begin{array}{c}
0_{n \times n} I_{n \times n} \\
-\Omega-Z
\end{array}\right], \quad B=\left[\begin{array}{c}
0_{n \times 1} \\
-\Phi^{T} K_{u v}
\end{array}\right],
$$

onde $A$ é a matriz do sistema e $B$ é a matriz de controle.

\section{Controle de Vibrações}

Após a modelagem e discretização da estrutura, iniciou-se a implementação do controle na viga. Neste trabalho se propõe a utilização do efeito do buracos prescritos como controle passivo e para o controle ativo, implementou-se um controlador ótimo realimentado que levará em conta um atuador piezoelétrico acoplado à viga.

\subsection{Controle Passivo}

A utilização do efeito dos buracos prescritos como controle passivo foi descrito pela primeira vez por Mironov (1988), denotado por ele como Buracos Negros Acústicos (BNAs). Seu princípio básico diz que não há reflexão de uma onda que se propaga em uma estrutura onde a espessura diminui suavemente. Isso se dá pelo fato de que, no momento em que a onda entra no buraco, tem sua velocidade reduzida e sua amplitude aumentada, consequentemente, a energia dessa onda é concentrada dentro deste buraco, conforme ilustrado na Fig. 2.

Para atender a condição da espessura, o perfil do buraco prescrito necessita seguir à uma lei de potência, ou seja, a espessura da estrutura precisa diminuir conforme:

$$
h(x)=a x^{m}+h_{1},
$$

no qual $a$ é uma constante que depende da largura e da altura do buraco prescrito, $m$ é a ordem do buraco, $x$ é comprimento da viga e $h_{1}$ é o resíduo de espessura dentro do buraco. $O$ termo $h_{1}$ na Eq. (19) se faz necessário devido ao problema de manufatura da prática, pois é impossível 


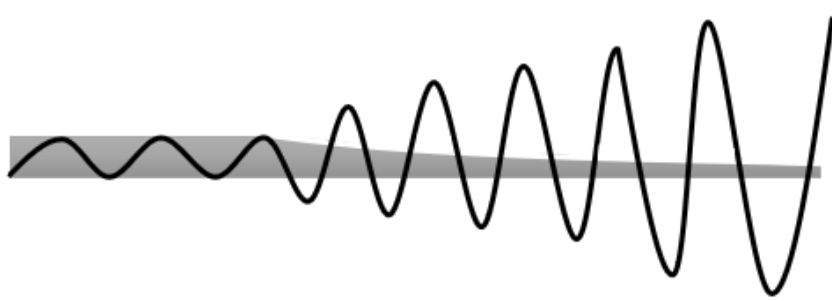

Figura 2: Efeito do buraco prescrito.

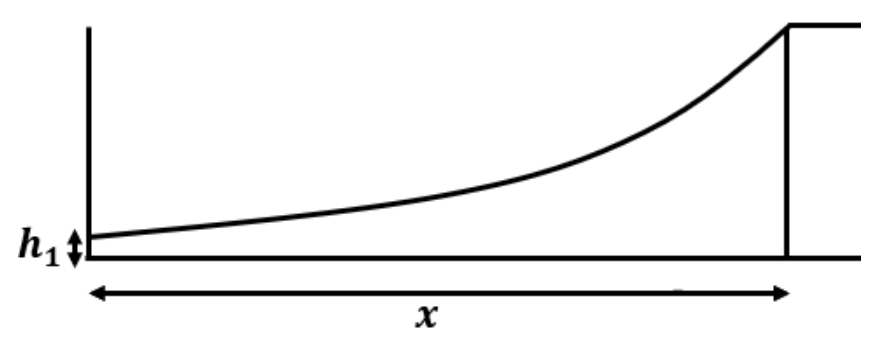

Figura 3: Formato de um buraco prescrito.

produzir um perfil que tenda a espessura à zero, restando sempre uma espessura residual (Zhao and Prasad, 2019).

O formato de um buraco com a espessura definida pela Eq. (19) está apresentado na seguinte imagem:

De acordo com Zhao and Prasad (2019), existem diversos fatores que influenciam na maneira como o buraco vai atuar na estrutura, entre eles a quantidade de buracos, 0 seu comprimento, a ordem $m$ do buraco, o tamanho do resíduo $h_{1}$ e a sua configuração na estrutura. No presente trabalho foram realizados diversos testes para se encontrar a melhor forma de aplicação do efeito dos buracos na estrutura.

\subsection{Controle Ativo}

Como controle ativo implementou-se um controlador ótimo realimentado, considerando o Regulador Quadrático Linear (LQR), que irá levar em conta um atuador piezoelétrico acoplado (colado) à viga.

De acordo com Hu et al. (2018), o controle LQR normalmente é empregado para determinar a realimentação. A matriz de ganho $G$ é escolhida de forma a minimizar o índice de desempenho $J$, na forma:

$$
J=\frac{1}{2} \int_{0}^{\infty}\left(\xi^{T} Q \xi+v_{a}^{T} R v_{a}\right) d t
$$

onde as matrizes $Q$ e $R$ são positivas semidefinidas e seus elementos são escolhidos para conceder um desempenho adequado. $O$ valor de $v_{a}$ pode ser encontrado pela seguinte Eq. (21):

$$
v_{a}=-G \xi
$$

Para determinar o ganho G na Eq. (21), basta resolver a

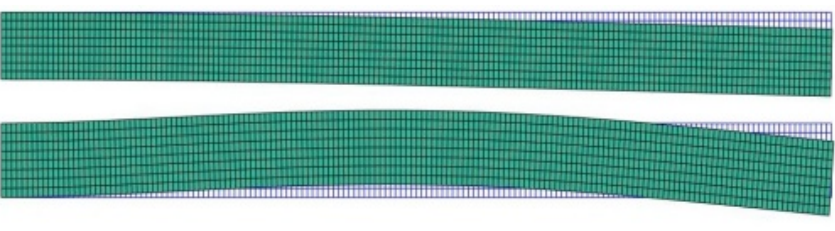

Figura 4: Primeiro e segundo modos obtidos da Eq. (10) Viga sem buraco e sem atuador.

Eq. (22):

$$
G=R^{-1} B^{T} P
$$

no qual a matriz $B$ é definida na Eq. (18), e $P$ é uma matriz auxiliar encontrada a partir da resolução da equação de Riccati:

$$
A^{T} P+P A-P B R^{-1} B^{T} P+Q=0,
$$

sendo que a matriz A também é definida na Eq. (18).

\section{Resultados e discussões}

Nesta seção serão realizadas duas análises. A primeira análise, Seção 5.1, apresentará as respostas de amplitude das configurações dos buracos prescritos utilizados na estrutura. Será analisado qual configuração apresenta uma menor amplitude de vibração, considerando os dois primeiros modos. Na segunda análise, Seção 5.2, irá se escolher a melhor configuração a partir da análise da Seção 5.1, ou seja, a melhor resposta de amplitude de vibração. Com isso será adicionado o atuador piezoelétrico à viga e aplicado o controle ativo à viga.

As simulações foram feitas através de códigos implementados no MATLAB, os gráficos da amplitude de vibração foram obtidos pela Transformada de Fourier. Foi usado uma malha com 160 elementos em $x$ e 8 elementos em $y$. 0 material considerado para as constantes constitutivas foi o Alumínio, com módulo de Young $\left(E_{e}\right)$ igual a $65 \times 10^{9} \mathrm{GPa}$, densidade $(\rho)$ igual a $2890 \mathrm{~kg} / \mathrm{m}^{3}$ e coeficiente de Poisson $(\nu)$ igual a 0,334 (Molter et al., 2010).

Na Fig. 4 estão representados os dois primeiros modos da viga sem buracos e sem o atuador piezoelétrico.

Na Fig. 5 é possível observar a resposta de amplitude de vibração da viga sem buraco prescrito e sem atuador piezoelétrico, considerando os dois primeiros modos.

\subsection{Análise de amplitude da vibração}

A primeira análise será feita com base na resposta de amplitude de vibração de cada uma das configurações dos buracos prescritos. A primeira configuração a ser analisada é da viga com um buraco. Os dois primeiros modos dessa viga são mostrados na Fig. 6 e sua resposta de amplitude é mostrada na Fig. 7.

A segunda configuração a ser analisada é a viga com dois buracos. O primeiro e o segundo modos dessa viga são mostradas na figura Fig. 8 abaixo.

A resposta de amplitude desta configuração conside- 


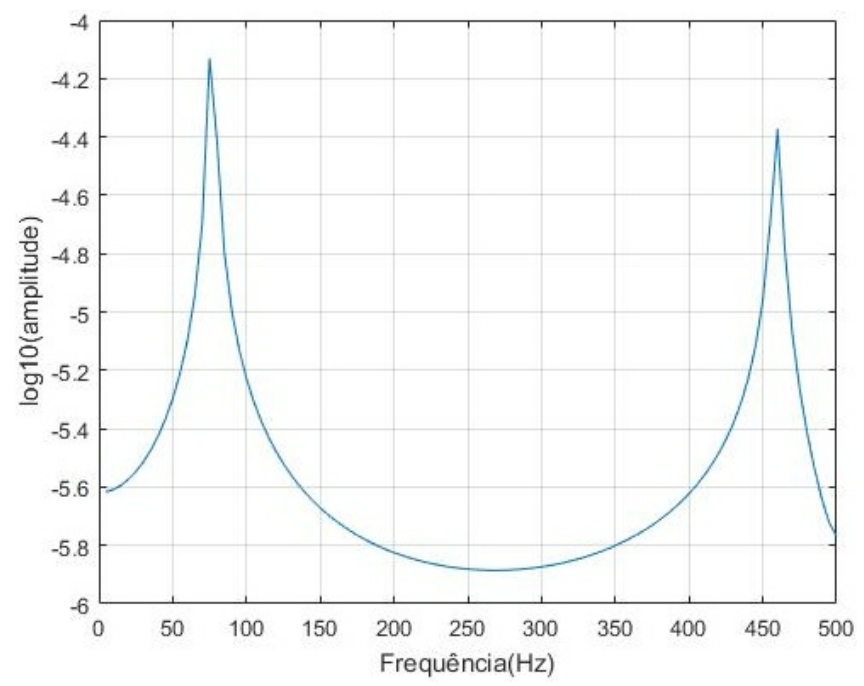

Figura 5: Resposta de amplitude da vibração - Viga sem buraco e sem atuador.

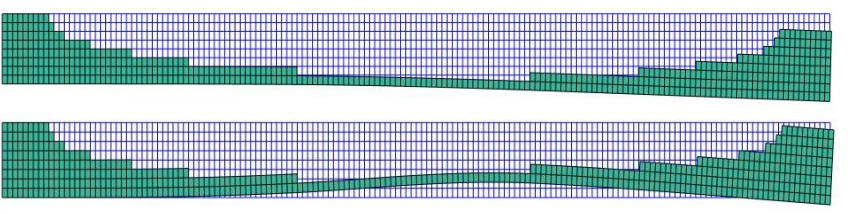

Figura 6: Primeiro e segundo modos obtidos da Eq. (10) Viga com um buraco e sem atuador.

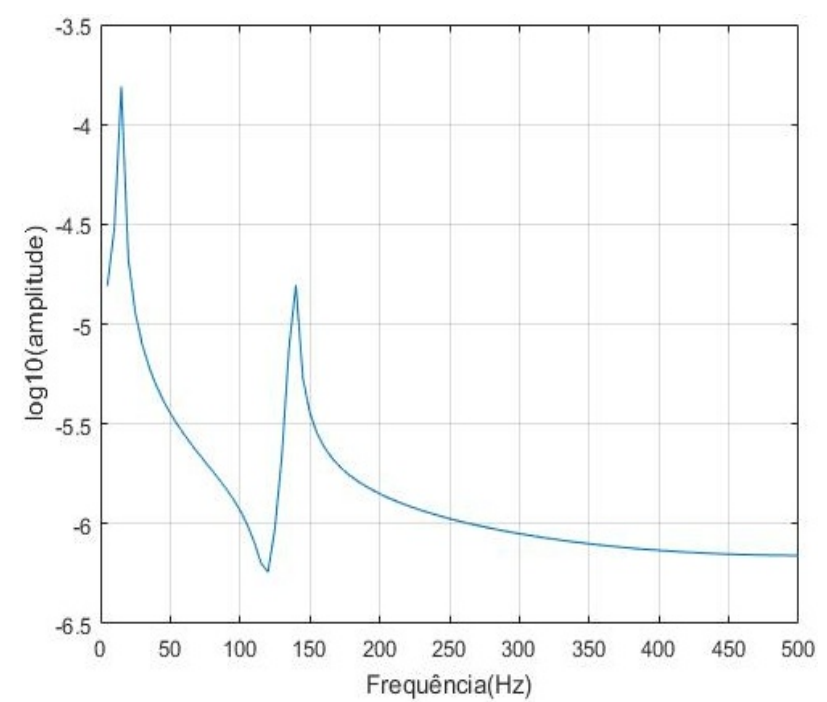

Figura 7: Resposta de amplitude da vibração - Viga com um buraco e sem atuador.

rando os dois primeiros modos é mostrada na Fig. 9.

A terceira e última configuração a ser testada é a da viga com três buracos. Os seus dois primeiros modos são mostrados na Fig. 10 e a resposta de amplitude na Fig. 11.

Neste trabalho usamos dois modos de vibração. Pela

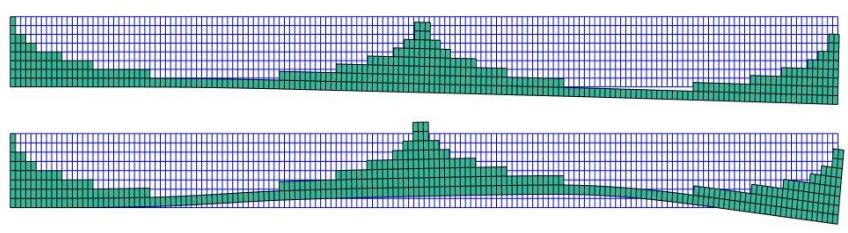

Figura 8: Primeiro e segundo modos obtidos da Eq. (10) Viga com dois buracos e sem atuador.

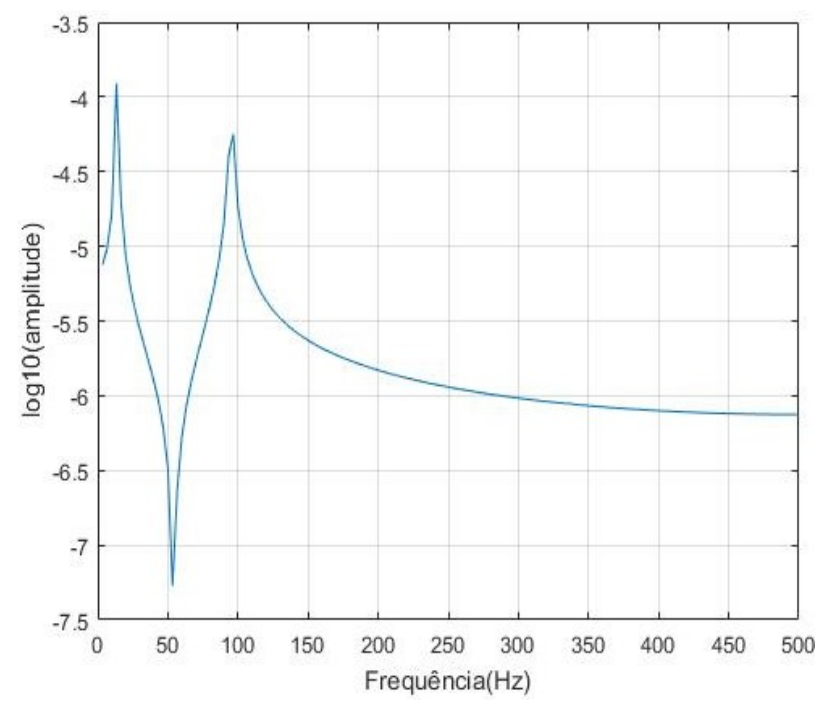

Figura 9: Resposta de amplitude da vibração - Viga com dois buracos e sem atuador.

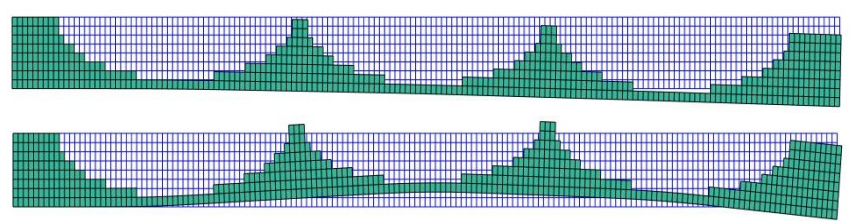

Figura 10: Primeiro e segundo modos obtidos da Eq. (10) Viga com três buracos e sem atuador.

técnica da superposição modal, o deslocamento final é resultante da soma dos deslocamentos modais (a soma é feita do resultado das amplitudes modais em metros). Sendo assim, as amplitudes modais nas Figs. 5, 7, 9 e 11 foram somados.

Abaixo segue uma tabela com os valores das somas das amplitudes máximas e suas duas primeiras frequências naturais para a viga sem buraco e para cada configuração de viga com buracos mostrada acima.

Pode-se observar que a configuração com um buraco demonstrou uma melhor resposta, já que teve uma amplitude máxima de deslocamento menor e também uma resposta de amplitude de vibração com decaimento maior com o aumento da frequência.

Os trabalhos de Zhao and Prasad (2019) e Pelat et al. (2020) indicam que o efeito dos buracos na estrutura pode ser mais efetivo para os modos de vibração de frequências 


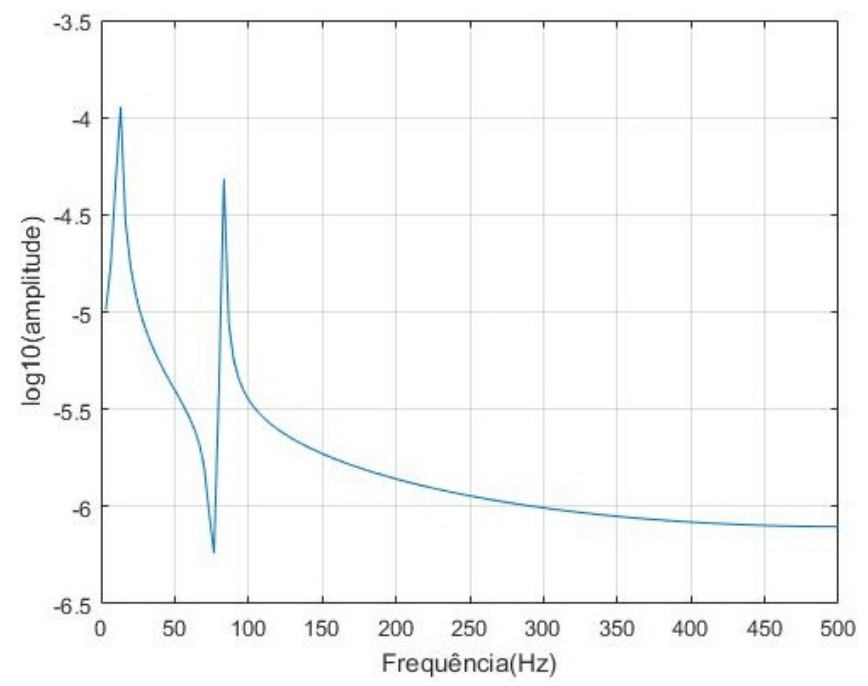

Figura 11: Resposta de amplitude da vibração - Viga com três buracos e sem atuador.

Tabela 1: Resultados dos testes com os buracos prescritos para o primeiro modo

\begin{tabular}{|l|l|l|l|}
\hline Quantidade de buracos & $\left|A_{\operatorname{máx}}\right|(\mathrm{m})$ & $\omega_{1}(\mathrm{~Hz})$ & $\omega_{2}(\mathrm{~Hz})$ \\
\hline Sem buraco & $5,2696 \cdot 10^{-4}$ & 76,25 & 457,28 \\
\hline 1 & $4,0799 \cdot 10^{-4}$ & 14,15 & 137,46 \\
\hline 2 & $5,0851 \cdot 10^{-4}$ & 13,74 & 94,93 \\
\hline 3 & $4,2901 \cdot 10^{-4}$ & 12,25 & 82,85 \\
\hline
\end{tabular}

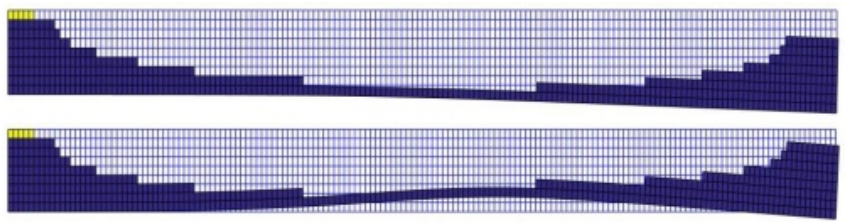

Figura 12: Primeiro e segundo modos - Viga com um buraco e atuadores piezoelétricos.

mais altas. Embora neste trabalho sejam considerados apenas dois modos de vibração, observou-se que já houve uma boa atenuação, o que mostra que o efeito dos buracos na atenuação de vibrações também depende da estrutura utilizada.

\subsection{Controle das vibrações}

Decidida a melhor configuração dentre um, dois ou três buracos, acoplou-se um atuador piezoelétrico na viga, de material PZT5A, considerando não haver perdas de propriedades mecânicas no acoplamento, com módulo de Young $\left(E_{a}\right)$ igual a $50 \times 10^{9} \mathrm{GPa}$ e densidade $(\rho)$ igual a $7600 \mathrm{~kg} / \mathrm{m}^{3}$ (Premount, 2011). E com as constantes constitutivas $c_{11}$ igual a $12,1 \times 10^{10}, c_{12}$ igual a $7,54 \times 10^{10}$ e $c_{66}$ igual a $2,26 \times 10^{10}$. (Molter et al., 2016). Para isso adicionou-se elementos acima da viga com as características de massa e rigidez do material piezoelétrico, conforme mostra a Fig. 12, para o primeiro e segundo modos, respectivamente.

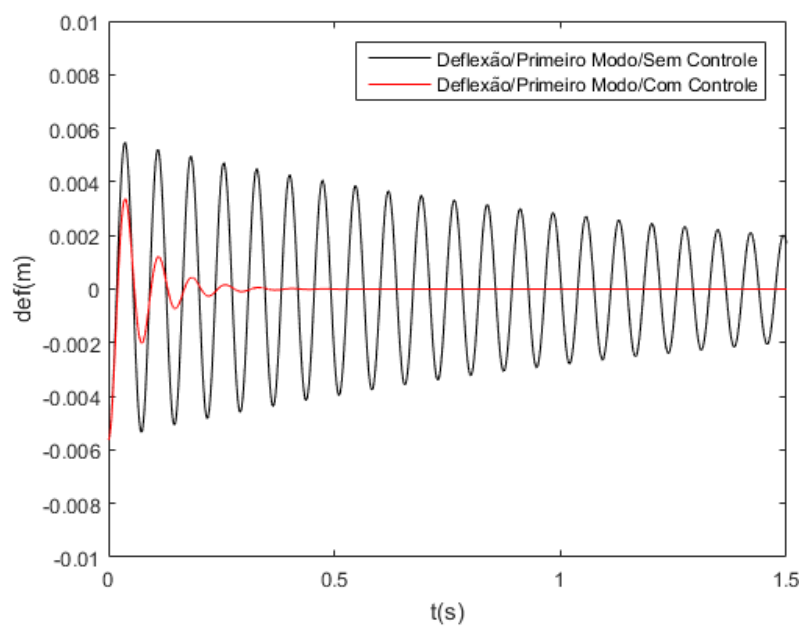

(a)

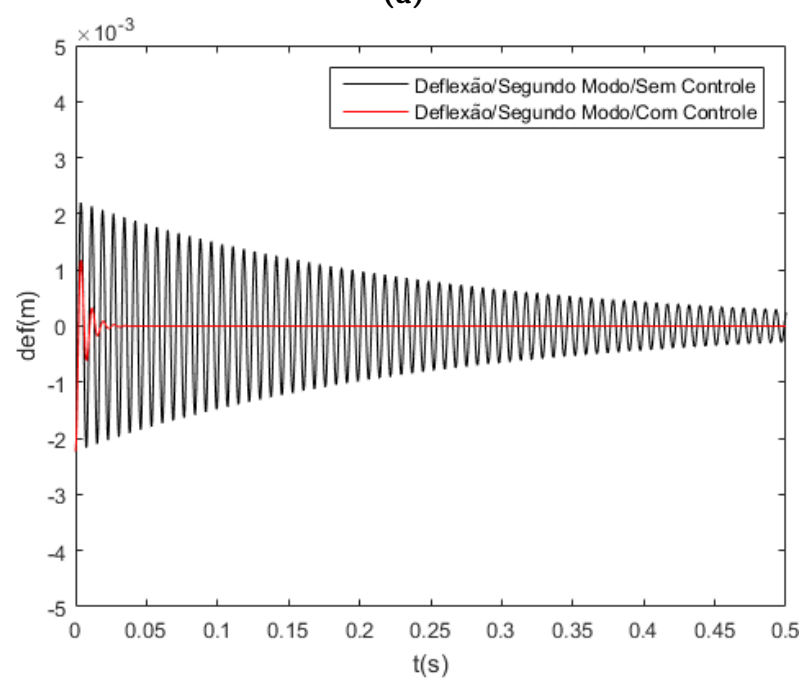

(b)

Figura 13: Movimento transversal do final da viga com um buraco prescrito e um atuador piezoelétrico com e sem controle para o primeiro modo (a) e segundo modo

(b), obtidos da Eq. (17).

Após isso, foi aplicado o controle à viga. Foi feito o controle para os dois modos separadamente para facilitar o projeto. A matriz $R$ utilizada em ambos os modos foi uma matriz identidade (I) com dimensão 2 vezes o número de nós dos elementos referentes ao material piezoelétrico. A matriz $Q$ utilizada também foi uma matriz identidade (I) porém com dimensão $2 \times 2$ em cada grau de liberdade considerado para o deslocamento transversal, multiplicada por $10^{12}$, para o primeiro modo, e por $10^{14}$, para o segundo modo. Considerou-se o deslocamento transversal para ambos os modos, no último nó da malha de Elementos Finitos. O movimento final dos dois primeiros modos são mostrados na Fig. 13.

Pode-se observar que, na Fig. 13, a amplitude inicial reduziu significativamente, também é possível notar que, em menos de 1 segundo, a vibração foi controlada, o que 
mostra que o controle LQR foi eficiente no controle das vibrações.

\section{Conclusões}

Este trabalho propôs o controle de vibrações em uma viga em balanço com buracos prescritos. Foi considerado simultaneamente um controle passivo, através de buracos nas vigas, e um controle ativo, através do acoplamento de atuadores piezoelétricos na estrutura.

A partir das duas técnicas de controle proposto, concluiu-se que a utilização dos buracos prescritos auxiliou no controle de vibrações da viga. Em relação ao controle ativo, o controlador LQR se mostrou eficiente. Como atuador, foi considerado o material piezoelétrico, o qual é bastante utilizado no controle de vibrações e confirmou-se sua efetividade.

Na comparação dos resultados deste trabalho com outros trabalhos, como os de O'Boy et al. (2010), Krylov (2012) e Zhao and Prasad (2019), pode-se perceber que o controle proposto foi satisfatório, embora esta comparação não seja a mais adequada, pois neles só foi considerado o amortecimento das vibrações utilizando os efeitos dos BNAs e as estruturas consideradas são diferentes das propostas no presente trabalho. Em relação aos atuadores, podemos verificar no trabalho de Schulz et al. (2013) que o resultado é similar ao obtido no presente artigo.

Como continuação desta pesquisa, pretende-se desenvolver uma metodologia para otimização da quantidade e geometria dos buracos prescritos.

\section{Agradecimentos}

A autora Tatiane Weimann agradece o apoio financeiro da Coordenação de Aperfeiçoamento de Pessoal de Nível Superior - Brasil (CAPES) - Código de Financiamento 001.

\section{Referências}

Becker, J., Fein, O., Maess, M. and Gaul, L. (2006). Finite element-based analysis of shunted piezoelectric structures for vibration damping, Computers \& Structures 84: 2340-2350. https://doi .org/10.1016/j . compstruc . 2006.08 .067$.

Chandran.P, S. and Thampan, C. P. V. (2017). A study on vibration control of structures due to seismic excitation using tuned mass damper, International Journal of Scientific \& Engineering Research 8: 105-112.

Dubay, R., Hassan, M., Li, C. and Charest, M. (2014). Finite element based model predictive control for active vibration suppression of a one-link flexible manipulator, ISA Transactions 53: 1609-1619. https://doi.org/10.1016/ j.isatra.2014.05.023.

Fuller, C. C., Elliot, S. J. and Nelson, P. A. (1996). Active Control of Vibration, 1 edn.

Gawronski, W. K. (2004). Advanced Structural Dynamics and Active Control of Structures, 1 edn, Springer, Berlin, NY.
Herrmann, C., Dewulf, W., Hauschild, M., Kaluza, A., Kara, S. and Skerlos, S. (2018). Life cycle engineering of lightweight structures, CIRP Annals - Manufacturing Technology 67: 651-672. https://doi.org/10.1016/j. cirp.2018.05.008.

Hiruta, T., Hosoya, N., Maeda, S. and Kajiwara, I. (2021). Experimental validation of vibration control in membrane structures using dielectric elastomer actuators in a vacuum environment, International Journal of Mechanical Sciences 191: 106049. https: //doi.org/10.1016/j . ijmecsci.2020.106049.

$\mathrm{Hu}$, J., Zhang, X. and Kang, Z. (2018). Layout design of piezoelectric patches in structural linear quadratic regulator optimal control using topology optimization, Intelligent Material Systems and Structures 29: 2277-2294. https://doi.org/10.1177/1045389X18758178.

Kelly, S. G. (2012). Mechanical Vibrations - Theory and Aplications, 1 edn, Christopher M. Shortt, Stamford, EUA.

Kras, A. and Gardonio, P. (2020). Active vibration control unit with a flywheel inertial actuator, Journal of Sound and Vibration 464: 114987. https: //doi .org/10.1016/j . jsv.2019.114987.

Krylov, V. V. (2012). Acoustic black holes and their applications for vibration damping and sound absorption, International conference on Noise and Vibration Engineering 1: 933-944. Disponível em https://repository. lboro.ac.uk/ndownloader/files/16802291/1.

Liu, G. R. and Quek, S. S. (2003). The finite element method: A practical course, 1 edn, Butterworth Heinemann, Burlington, EUA.

Mironov, M. A. (1988). Propagation of a flexural wave in a plate whose thickness decreases smoothly to zero in a finite interval, 34: 546--547.

Mohammadrezazadeh, S. and Jafari, A. A. (2020). Active vibration control of rotating laminated composite truncated conical shells through magnetostrictive layers based on first-order shear deformation theory, Journal of the Brazilian Society of Mechanical Sciences and Engineering 42: 304. https://doi.org/10.1007/ s40430-020-02363-w.

Molter, A., Fernandez, L. d. S. and Lauz, J. B. (2018). An optimality criteria-based method for the simultaneous optimization of the structural design and placement of piezoelectric actuators, Structural and Multidisciplinary Optimization 59: 1125-1141. https://doi.org/10.1007/ s00158-018-2116-y.

Molter, A., Fonseca, J. S. O. and Fernandez, L. d. S. (2016). Simultaneous topology optimization of structure and piezoelectric actuators distribution, Applied Mathematical Modelling 40: 5576-5588. http://dx.doi.org/10. 1016/j.apm.2016.01.023.

Molter, A., Silveira, O. A. A. d., Fonseca, J. S. O. and Bottega, V. (2010). Simultaneous piezoelectric actuator and sensor placement optimization and control design of manipulators with flexible links using sdre method, 
Mathematical Problems in Engineering 2010: 1-24. https : //doi.org/10.1155/2010/362437.

Nicoletti, R. (2013). Estudo do controle ativo e passivo de vibrações em sistemas rotativos e estruturais, Livre docência, Escola de Engenharia de São Carlos. Available at https: //doi.org/10.11606/T.18.2013.tde-29112013-102028.

O'Boy, D. J., Krylov, V. V. and Kralovic, V. (2010). Damping of flexural vibrations in rectangular plates using the acoustic black hole effect, Journal of Sound and Vibration 329: 4672-4688. https://doi . org/10.1016/j.jsv . 2010.05.019.

Ogata, K. (2014). Engenharia de Controle Moderno, 5 edn, Roger Trimer, São Paulo, Brasil.

Peña, L. A. P. (2017). Resposta dinâmica de edificações adjacentes acopladas: considerações sobre a interação solo - estrutura, Tese de doutorado, Faculdade de Tecnologia. Departamento de Engenharia Civil e Ambiental. Available at https://repositorio.unb.br/handle/10482/23371.

Pelat, A., Gautier, F., Conlon, S. C. and Semperlotti, F. (2020). The acoustic black hole: A review of theory and applications, Journal of Sound and Vibration 476: 115316. https://doi.org/10.1016/j.jsv.2020.115316.

Premount, A. (2011). Vibration Control of Active Structures - An Introduction, 3 edn, Springer Netherlands, Países Baixos.

Ramezani, M. and Labafzadeh, M. (2021). Passive and semi-active vibration control of base- isolated structure under blast loading at medium to long distances, Amirkabir (Journal of Science and Technology) .

Schulz, S. L., Gomes, H. M. and Awruch, A. M. (2013). Optimal discrete piezoelectric patch allocation on composite structures for vibration control based on ga and modal lqr, Computers and Structures 128: 101-115. https : //doi.org/10.1016/j. compstruc. 2013.07.003.

Tian, J., Guo, Q. and Shi, G. (2020). Laminated piezoelectric beam element for dynamic analysis of piezolaminated smart beams and ga-based lqr active vibration control, Composite Structures 252: 112480. https: //doi.org/10.1016/j. compstruct. 2020.112480.

Zhao, C. and Prasad, M. G. (2019). Acoustic black holes in structural design for vibration and noise control, Acoustics 1: 220-251. https://doi.org/10.3390/ acoustics1010014. 